\title{
Photostability of Fullerene and Non-Fullerene Polymer Solar Cells: The Role of the Acceptor
}

\author{
Nutifafa Y. Doumon,*๑ Mikhail V. Dryzhov, Félix V. Houard, Vincent M. Le Corre, \\ Azadeh Rahimi Chatri, $\odot$ Panagiotis Christodoulis, and L. Jan Anton Koster*๑
}

Photophysics and Optoelectronics, Zernike Institute for Advanced Materials, University of Groningen, Nijenborgh 4, NL-9747 AG, Groningen, The Netherlands

\section{Supporting Information}

ABSTRACT: Recently, the advent of non-fullerene acceptors (NFAs) made it possible for organic solar cells (OSCs) to break the $10 \%$ efficiency barrier hardly attained by fullerene acceptors (FAs). In the past five years alone, more than hundreds of NFAs with applications in organic photovoltaics (OPVs) have been synthesized, enabling a notable current record efficiency of above $15 \%$. Hence, there is a shift in interest toward the use of NFAs in OPVs. However, there has been little work on the stability of these new materials in devices. More importantly, there is very little comparative work on the photostability of FA versus NFA solar cells to ascertain the pros and cons of the two systems. Here, we show the photostability of solar cells based on two workhorse acceptors, in both conventional and inverted structures, namely, ITIC (as NFA) and [70]PCBM (as FA), blended with either

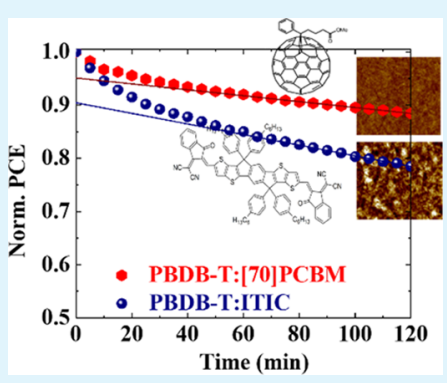
PBDB-T or PTB7-Th polymer. We found that, irrespective of the polymer, the cell structure, or the initial efficiency, the [70]PCBM devices are more photostable than the ITIC ones. This observation, however, opposes the assumption that NFA solar cells are more photochemically stable. These findings suggest that complementary absorption should not take precedence in the design rules for the synthesis of new molecules and there is still work left to be done to achieve stable and efficient OSCs.

KEYWORDS: Fullerene derivatives, Non-Fullerene acceptors, Degradation, Organic solar cells, Photostability

\section{INTRODUCTION}

Organic solar cell (OSC) technologies have evolved over the years in terms of architecture, processing techniques, and most especially the semiconductor materials used in the active layers. The bulk heterojunction $(\mathrm{BHJ})$ has proven so far to be the best configuration for the active layer. The active layer under this configuration is an interconnecting network of donor (D) and acceptor (A) molecules, selected such that there is an appropriate LUMO offset in their energy levels, thus facilitating in part easy pathways for charge carrier extraction. These molecules are usually polymers, small molecules, and/or fullerene derivatives. The choice of these materials is crucial to the performance of the devices in terms of efficiency and stability. Previous works ${ }^{1-15}$ have been industrious in improving the efficiency of organic semiconductor devices, especially in the case of OSCs in tuning the donor material's compatibility with the fullerene acceptors (FA), mostly [70]PCBM with an efficiency rarely reaching beyond $10 \% .^{12,13,16-18}$ However, because of the limited light absorption of the fullerene derivatives in the visible range of the solar spectrum, coupled with limitations in their energy level tunability, the performance of OSCs has hit a bottleneck. Scientists found alternatives in either polymers in what they termed as "all polymer" solar cells ${ }^{19-22}$ or small molecules, which they referred to as "non-fullerene/fullerene-free" solar cells ${ }^{4,23-29}$ [e.g., 3,9-bis (2-methylene-(3-(1,1-dicyanomethylene)-indanone))-5,5,11,11-tetrakis(4-hexylphenyl)-dithieno- $\left[2,3-d: 2^{\prime}, 3^{\prime}-d^{\prime}\right]-s$-indaceno $\left[1,2-b: 5,6-b^{\prime}\right]$ dithiophene, ITIC, based small molecule derivatives]. These novel acceptors have boosted the power conversion efficiency (PCE) of OSCs up to above $14 \%$ in single junction ${ }^{30-32}$ and $17.3 \%$ for multijunction ${ }^{33} \mathrm{BHJ}$ solar cells. This is made possible because of the acclaimed properties of the non-fullerene acceptors (NFA), namely, their easy synthesis, strong absorption, tunable properties, and enhanced stability. ${ }^{34}$

While this new development is exciting, it is important to point out that studies on device stability and its subsequent improvement are lagging far behind. Recently, there have been notable works on studying the stability of the best-performing donor polymers blended with [70] PCBM. ${ }^{5,35-43}$ With the advent of the NFAs currently outperforming FAs, the focus has been on the device efficiency and little is done to understand their device stability. ${ }^{29,31,44-46}$ It has been suggested that current NFAs, for example, ITIC and its derivatives, can be more thermally and photochemically stable than FAs, ${ }^{29,34}$ but there have been no to a few studies to ascertain this assumption. For example, IDTBR NFAs among others are shown to be more stable. ${ }^{25,47,48}$ Even in such studies, the solar cells are considered under their presumed optimal conditions; that is, the FA-based cells are processed with additives

Received: November 21, 2018

Accepted: January 31, 2019

Published: January 31, 2019 
(namely, 1,8-diiodooctane, DIO)..$^{47,48}$ It is known (and as shown in the Supporting Information) that DIO negatively affects photostability. ${ }^{43,49,50}$ Thus, a necessary prerequisite and strong comparative study must consider both FA and NFA solar cells under comparable optimum conditions without the use of additives to understand the reason behind (i) the photochemical degradation and (ii) differences in the degradation pathways of FA and NFA solar cells in a systematic way.

In this work, the role of the acceptors in the photodegradation of their respective solar cells is explored under 1 sun illumination at a constant temperature by active cooling in an inert atmosphere in a glovebox, with both $\mathrm{O}_{2}$ and $\mathrm{H}_{2} \mathrm{O}$ levels kept below $0.1 \mathrm{ppm}$. Thus, a comparative photostability study between a fullerene derivative acceptor, [70]PCBM, and the widely used non-fullerene small molecule acceptor, ITIC, is performed in blends with the PBDB-T polymer. A second polymer PTB7-Th, the famous BDT-TT polymer (also known as PCE10), is used to corroborate the findings (see Figure S2). The choice of acceptors is purely based on the fact that they are commercially available and are workhorse materials in their own category. All studied materials are shown in Figure 1 with

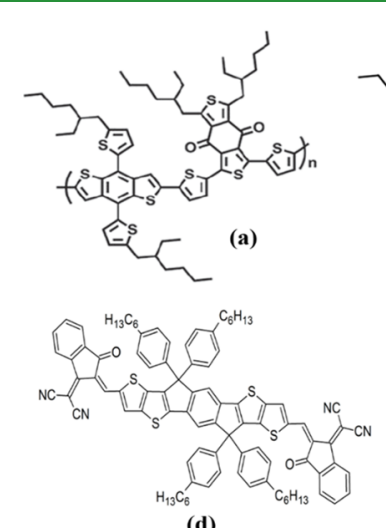

(d)

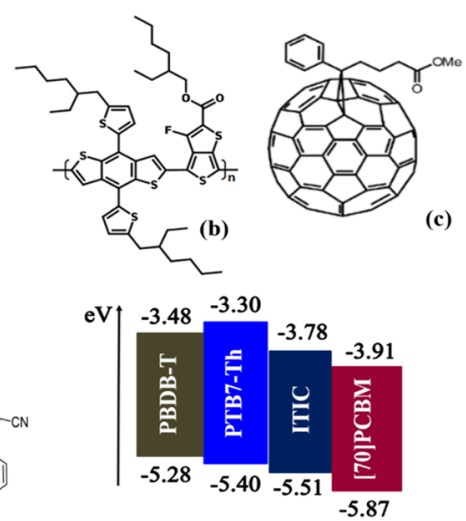

(e)
Figure 1. Chemical structures of PBDB-T (a), PTB7-Th (b), [70]PCBM (c), and ITIC (d) with their energy level diagrams (e).

their energy levels. The photoinduced degradation behaviors of the devices based on the two acceptors are studied via device physics with a combination of measurement techniques such as current-voltage characterization for monitoring changes in efficiency, charge transport, and recombination processes; UV-vis-NIR absorption for tracking changes in absorption; atomic force microscopy (AFM) for detecting changes in morphology; and transient photovoltage (TPV) together with extraction measurement for monitoring changes in (ratio of) rates of recombination and extraction. With these techniques, the differences in performance, both in PCE and photostability, of the small molecule acceptor (NFA) and the fullerene derivative [70]PCBM (FA) are elucidated, and the main reasons behind their instability are revealed.

\section{RESULTS AND DISCUSSION}

2.1. Performance: Power Conversion Efficiency. Conventional solar cells, with active layers processed with and without DIO, were fabricated as described in detail under the section Experimental Procedures. Their current-voltage characteristics were monitored under continuous 1 sun illumination over time at a constant temperature of $295 \mathrm{~K}$ by active cooling in an inert atmosphere in a glovebox, with both $\mathrm{O}_{2}$ and $\mathrm{H}_{2} \mathrm{O}$ levels kept below $0.1 \mathrm{ppm}$, to evaluate their performance in terms of efficiency and photostability. Some of the devices were processed with DIO (3 vol \% for the [70]PCBM-based devices and 0.5 vol $\%$ for the ITIC-based devices). DIO is known to help improve the efficiency of OSCs. The best-performing PBDB-T:[70]PCBM solar cells yielded $7.1 \%$ without DIO and $7.8 \%$ with DIO in PCE, while the best-performing PBDBT:ITIC solar cells recorded $8.1 \%$ without DIO and $7.6 \%$ with DIO. For devices without DIO, the ITIC-based cells have outperformed the [70]PCBM-based ones in efficiency, mainly because of the increment in the short-circuit current density $J_{\text {sc. }}$ The same trend is observed for inverted solar cells with $8.6 \%$ for ITIC-based solar cells and $5.7 \%$ for [70] PCBM-based ones. The best current-voltage parameters and average values of the PCEs are displayed in Figure $2 \mathrm{a}$ and Table 1, while the count of PCE of all fabricated
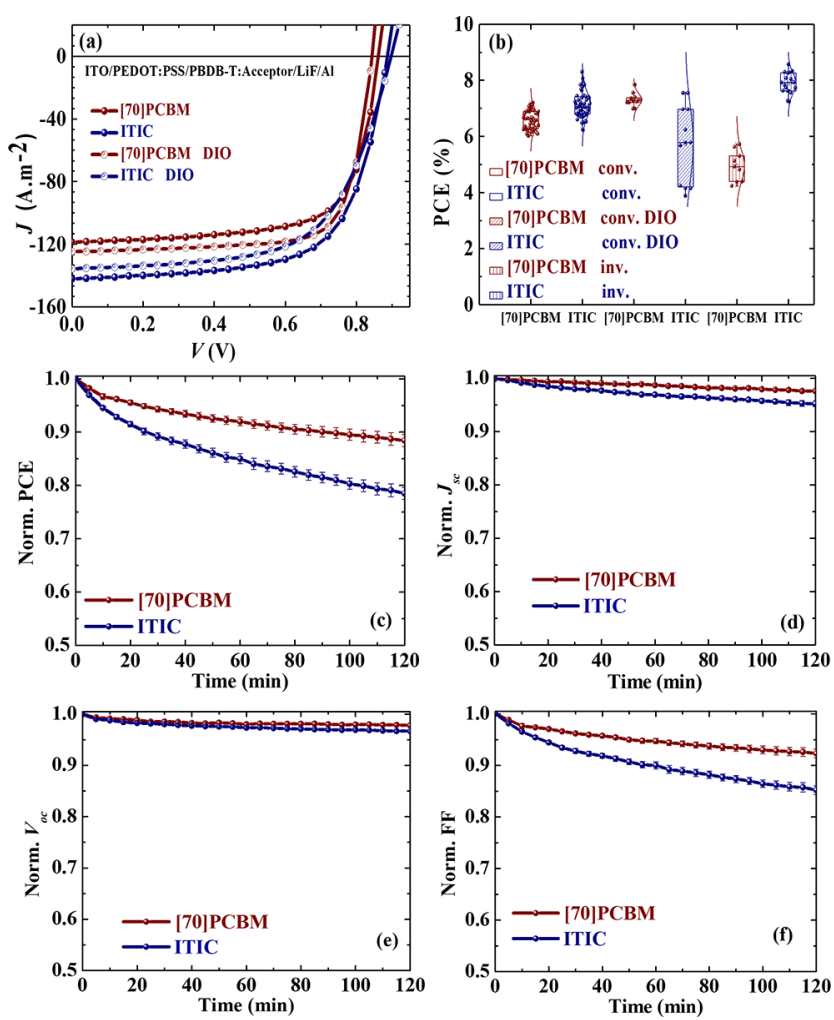

Figure 2. Performance of PBDB-T-based solar cells: current-voltage curves of best conventional solar cells with/without DIO (a); device PCE statistics showing the PCE distribution with device structure and total number of devices (b); evolution of current-voltage parameters normalized to their initial values (at $t=0 \mathrm{~min}$ ) under continuous illumination (mean of about 20 devices each): PCE (c), $J_{s c}(\mathrm{~d}), V_{\mathrm{oc}}$ (e), and FF (f). The main loss in PCE is due to a loss in FF. conv., conventional; inv., inverted.

devices is shown in Figure $2 \mathrm{~b}$ with more than 50 conventional PBDB-T-based devices (without DIO) for each type. As shown in the Supporting Information and explained by recent reports, ${ }^{29,51}$ this difference in performance is partly due to the difference in the complementarity of the absorption of the $\mathrm{D}$ and the A materials: PBDBT has an overlapping spectrum with [70]PCBM, while it is complementary to the ITIC spectrum in the visible range. There is a $100 \mathrm{~nm}$ redshifted difference between the spectra of the two blends as seen in 
Table 1. Device Parameters of Cells under Study with Mean Values for the Cells Obtained for the Indicated Number of Devices per Type Processed either from CB or from CB:DIO

\begin{tabular}{|c|c|c|c|c|c|c|}
\hline device & treatment $^{a}$ & $L^{b}(\mathrm{~nm})$ & $J_{\mathrm{sc}}\left(\mathrm{A} \mathrm{m}^{-2}\right)$ & $V_{\text {oc }}(\mathrm{V})$ & FF (\%) & $\mathrm{PCE}\left(\mathrm{PCE}_{\mathrm{mn}} \pm \mathrm{SD}\right)^{c}(\%)$ \\
\hline PBDB-T:ITIC ${ }^{d}$ & conv., $100{ }^{\circ} \mathrm{C}, 10 \mathrm{~min}$ & 100 & 142.0 & 0.887 & 65.9 & $8.1(7.1 \pm 0.5)$ \\
\hline PBDB-T:ITIC & conv., $\mathrm{DIO}, 100{ }^{\circ} \mathrm{C}, 10 \mathrm{~min}$ & 100 & 135.7 & 0.898 & 62.0 & $7.6(5.8 \pm 1.4)$ \\
\hline PBDB-T:[70] PCBM $^{f}$ & conv. & 100 & 118.1 & 0.870 & 69.9 & $7.1(6.6 \pm 0.3)$ \\
\hline PBDB-T:[70]PCBM ${ }^{g}$ & conv., DIO & 100 & 124.7 & 0.844 & 74.5 & $7.8(7.3 \pm 0.2)$ \\
\hline PBDB-T:ITIC ${ }^{h}$ & inv., $160^{\circ} \mathrm{C}, 10 \mathrm{~min}$ & 100 & 145.0 & 0.831 & 71.1 & $8.6(8.1 \pm 0.4)$ \\
\hline PBDB-T:ITIC ${ }^{i}$ & inv., $\mathrm{DIO}, 160{ }^{\circ} \mathrm{C}, 10 \mathrm{~min}$ & 100 & 135.2 & 0.687 & 58.7 & $5.5(4.7 \pm 0.6)$ \\
\hline PBDB-T:[70] PCBM $^{j}$ & inv. & 100 & 112.6 & 0.842 & 60.4 & $5.7(5.0 \pm 0.5)$ \\
\hline PBDB-T:[70] PCBM $^{k}$ & inv., DIO & 100 & 128.6 & 0.765 & 64.5 & $6.4(6.3 \pm 0.04)$ \\
\hline
\end{tabular}

${ }^{a}$ conv.: conventional; inv.: inverted. ${ }^{b}$ L: thickness. ${ }^{c}$ mn: mean. ${ }^{d} 53$ devices. ${ }^{e} 12$ devices. ${ }^{f} 53$ devices. ${ }^{g} 12$ devices. ${ }^{h} 19$ devices. ${ }^{i} 7$ devices. ${ }^{j} 9$ devices. $k_{3}$ devices.

Figure S1a,b. Not only does the PBDB-T:ITIC film absorb more in the visible range than PBDB-T:[70] PCBM, but it also strongly absorbs at longer wavelengths, especially up to 800 $\mathrm{nm}$ in the IR region. Next, the better performance of the ITIC devices may also be explained by less trap-assisted recombination in their fresh devices compared to the [70]PCBM-based fresh devices. Indeed, as derived from the $V_{\text {oc }}$ light intensity dependence measurements in Figure S4, an ideality factor $(n)$, the lowest at 1.11 , is obtained for ITIC devices, while 1.34 is recorded for [70]PCBM devices. $n \frac{K T}{q}$, the slope of the $V_{\text {oc }}$ against varying light intensity, is a signature of the recombination mechanisms present in a solar cell. If $1<n<$ 2 , then trap-assisted recombination is dominant. ${ }^{52}$ However, an $n$ of $\sim 1$ means bimolecular recombination is dominant.

On the other hand, when PBDB-T is replaced in the blends by PTB7-Th, the opposite trend is observed as clearly shown by the current-voltage parameters in Table S1. That is more current from [70]PCBM-based devices as compared to ITIC ones. This observation is partly due, in this instance, to the complementarity of the absorption spectra of [70]PCBM and PTB7-Th and possibly due to the reduction in $n$ for the [70]PCBM devices with a value around 1.1. . $^{36,47}$

Turning to devices processed with DIO, the efficiency of PBDB-T:ITIC devices reduced, while that of PBDB-T: [70]PCBM increased. There is an increase in absorption intensity for [70]PCBM-based devices upon addition of DIO, as shown in Figure S1c. This results in higher $J_{\text {so }}$ suggesting a better balance in mobility and a smaller $n$ of 1.23 compared to 1.34 of the [70]PCBM-based devices without DIO. Next, ITIC-based devices with diiodooctane exhibited the complete opposite effect with a reduction in absorption peaks (see Figure S1d), with no apparent change in the ideality factor (1.09). As a result, they exhibited lower short-circuit current density. This partly explains the lower PCE of the ITIC devices. The current-voltage parameters are displayed in Table 1. Overall, the reported efficiencies especially for the ITIC-based devices are relatively lower than those reported in the literature ${ }^{29}$ because of differences in device fabrication conditions. However, these initial efficiencies do not affect the degradation behaviors.

2.2. Performance: Degradation and Stability. Figure 2 and Figure S2 display the degradation curves of the currentvoltage parameters of conventional solar cells without the additive (DIO) fabricated in an inert environment and measured in a glovebox with $\mathrm{H}_{2} \mathrm{O}$ and $\mathrm{O}_{2}$ levels below 0.1 ppm. Figure 3 displays the degradation curves of the currentvoltage parameters of inverted solar cells without DIO. Finally,
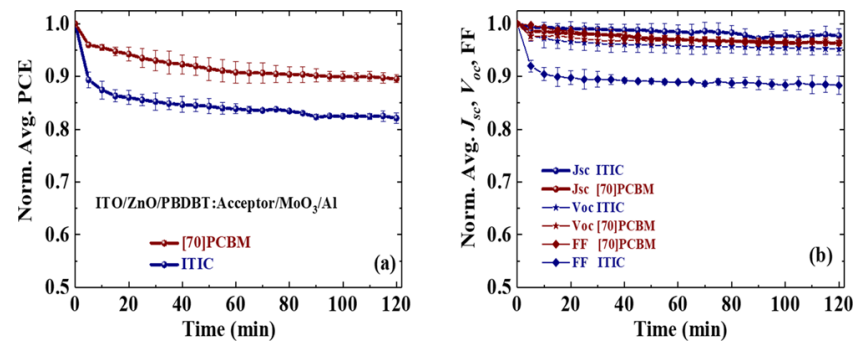

Figure 3. Performance under continuous illumination of PBDB-Tbased inverted solar cells (average of three devices): average PCE (a) and $J_{s c}, V_{\text {oc }}$ FF (b). Also, in here, the main loss is due to FF especially for the ITIC devices as can be seen.

Figure S3 displays the curves of conventional solar cells with DIO. The PCE degradation curves in Figure $2 \mathrm{c}$ reveal the same trend for all types of devices, thus a gradual decay (which gets accelerated in the presence of DIO in Figure S3) of the efficiency that slows down over time, with the ITIC devices losing more in PCE compared to the [70]PCBM ones. This is indicative of the role played by the donor and the acceptors in the degradation process. It is observed that ITIC-based devices are less stable than [70]PCBM-based ones. Among the current-voltage parameters, the FF accounts for the most loss in the PCE decay. The finding that ITIC-based devices are less stable than [70]PCBM-based ones is complementary to the works by Cha et al. and Baran et al., ${ }^{47,48}$ where they found that the EH-IDTBR NFA-based devices are more stable than the [70]PCBM-based ones. Thus, different NFA acceptor molecules may show different behaviors. It is worth noting that, in the study by Cha et al., a lamp without UV was used during the exposure time and all considered devices in the two studies were under their optimal conditions. ${ }^{48}$ Under such conditions, the [70]PCBM devices were processed with DIO, while the NFA ones were not. If this is the case, then two factors in addition to the difference in molecules would explain their observation, notably the absence of UV irradiation and the absence of DIO in one type of device. However, the observation is consistent with our devices with DIO, shown in Figure S3a. The difference in decay curves is acceptor dependence and so could be linked to D:A compatibility. In the case of devices with DIO, it could be due to the photoacidity of DIO and the formation of HI that could, in turn, react with the donor or the acceptor (or even both) materials as previously reported. These effects lead to a more rapid PCE decay. Additionally, in the case of ITIC-based devices with DIO, the changes in morphology also play a critical role in the degradation pathways. Thus, bigger losses in 
the current $\left(J_{s c}\right.$ in Figure S3c) and FF (in Figure S3b, a loss more pronounced in the presence of DIO) for ITIC devices were observed.

A closer look at the curves can only suggest the attenuation of the degradation (i) by the polymer structural modification (thus, changes in the backbone structure), (ii) by the acceptors, (iii) by the interaction/compatibility of both $\mathrm{D}$ and $\mathrm{A}$, and/or (iv) by the reduction in mobility over time. The first option cannot be the case as it would surely reflect in the same degradation pathway and strength because the same polymers are used with each of the acceptors. On the contrary, in Figure 2c, PBDB-T:ITIC exhibited on average 22\% PCE decay compared to PBDB-T:[70]PCBM, which recorded $12 \%$. Similar trends were observed in Figure $3 \mathrm{a}$ for inverted solar cells with an average of $19 \%$ decay for ITIC-based cells and $10 \%$ decay for [70]PCBM-based cells. Also, for PTB7-Thbased conventional cells in Figure S2a, ITIC cells showed 38\% decay in PCE compared to 9\% decay for [70]PCBM cells. For cells processed with DIO, PBDB-T:ITIC shows 70\% decay compared to the $40 \%$ of PBDB-T:[70]PCBM cells. This suggests that the acceptors play different roles in the acceleration or stabilization of the photodegradation. Thus, the acceptors themselves could play the role as stabilizers, or perhaps it is their intricate compatibility with the donor materials that slows down the photodegradation. To elucidate this point, absorption, AFM, charge transport, TPV, and extraction measurements were performed.

Photobleaching could be one of the reasons for the degradation of the polymer that results in the disruption of the $\pi$-conjugation. Absorption spectra of fresh and exposed blend films presented in Figure Sle,f show no significant changes over the period of exposure $(2 \mathrm{~h})$, explaining why we have observed almost no changes in $J_{\text {sc }}$. Next, AFM was used to check the changes in surface morphology upon light exposure. The results show no apparent changes in the morphology of the blend films without DIO in Figure 4. The surface

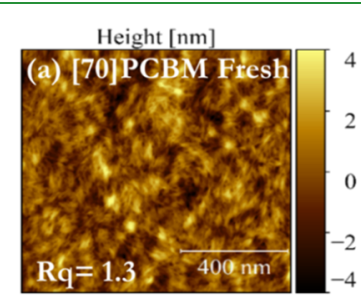

Height $[\mathrm{nm}]$

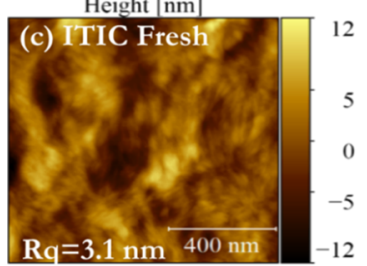

Height [nm]

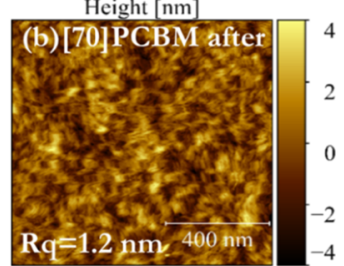

Height $[\mathrm{nm}]$

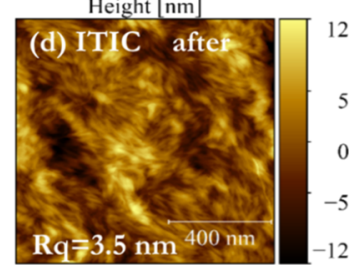

Figure 4. AFM images on the $1 \mu \mathrm{m}$ scale. PBDB-T:[70]PCBM cells: fresh (a) and exposed (b); PBDB-T:ITIC cells: fresh (c) and exposed (d).

roughness before (Figure 4a) and after the exposure (Figure $4 \mathrm{~b}$ ) is about $1.3 \mathrm{~nm}$ for [70]PCBM-based devices on the $1 \mu \mathrm{m}$ scale. The ITIC-based films under irradiation show no real changes in roughness on the same scale, with roughness from 3.1 (Figure 4c) to $3.5 \mathrm{~nm}$ (Figure 4d); however, the films seem to have become a bit more fibrillar. Even so, with these small changes, the observed degradation in the devices can be considered not mainly due to nanomorphological changes.

Single carrier devices of both pristine and blend materials are fabricated to check the changes in electron and hole transport before and after exposure to light. Insights into changes in mobility (a factor exhibited by the intricate compatibility between the donor and acceptor materials) could be linked to the difference in device degradation. The degree of changes in mobility may be affected by the different interfaces used between the transport layer and/or the electrodes. To avoid this effect in the exposed devices, the active layers are degraded/exposed to light before the top electrode evaporation. The resulting current-voltage curves are presented in Figure 5. Figure 5a,b presents the hole and electron current of pristine materials before and after exposure, and the derived mobilities from the space charge limited current (SCLC) method are presented in Table 2. The electron currents of pristine ITIC and [70]PCBM (not shown here, but shown in our previous work ${ }^{36}$ ) show no change before and after light exposure, suggesting no observable degradation of the acceptor materials. However, a tiny decrease in hole current was observed for PBDB-T, resulting in a decrease in hole mobility of the pristine PBDB-T from $10 \times 10^{-5}$ to $6 \times 10^{-5} \mathrm{~cm}^{2} \mathrm{~V}^{-1}$ $\mathrm{s}^{-1}$. These observations suggest that PBDB-T degrades under light exposure, while [70]PCBM and ITIC do not. Similar degradation is observed in one of our earlier studies ${ }^{36}$ for pristine PTB7-Th. However, it is more pronounced.

PBDB-T:[70]PCBM single carrier devices showed decreases in electron and hole currents in Figure 5e,f, respectively, reducing the electron mobility of the blend from $2.7 \times 10^{-4}$ to $6.3 \times 10^{-5} \mathrm{~cm}^{2} \mathrm{~V}^{-1} \mathrm{~s}^{-1}$ and the hole mobility from $10 \times 10^{-5}$ to $4.5 \times 10^{-5} \mathrm{~cm}^{2} \mathrm{~V}^{-1} \mathrm{~s}^{-1}$. As the decrease in electron mobility was more significant, it resulted in more balanced charge mobilities as depicted by the reduction in the ratio of mobilities $\left(\mu_{\max } / \mu_{\min }\right)$ from 2.7 toward unity (1.4). Thus, charge transport could become more balanced in [70]PCBMbased devices after light exposure. On the contrary, while the hole current of ITIC-based blend remains almost constant in Figure $5 \mathrm{c}$, resulting in hole mobilities from $15 \times 10^{-5}$ to $17 \times$ $10^{-5} \mathrm{~cm}^{2} \mathrm{~V}^{-1} \mathrm{~s}^{-1}$, the electron current shows a decrease (see Figure $5 \mathrm{~d}$ ), leading to a reduction in electron mobility from 5.1 $\times 10^{-5}$ to $2.8 \times 10^{-5} \mathrm{~cm}^{2} \mathrm{~V}^{-1} \mathrm{~s}^{-1}$. Such a decrease increases $\mu_{\max } / \mu_{\min }$ further away from unity from 2.9 to 6.1 . As a result, there is an imbalance in charge mobilities. This could mean that some charges remain in the device, forming an undesirable space charge that may oppose the flow of new charge carriers, leading to less charge extraction during the exposure time (photodegradation) and influencing the FF. This may explain why [70]PCBM-based solar cells are more photostable over time than the ITIC-based ones.

One reason for the reduction in electron currents of the blends is attributed to the formation of radical species in the active layer upon light exposure that acts as electron traps, increasing trap-assisted recombination. ${ }^{36}$ To further investigate this point, light intensity dependence $V_{\text {oc }}$ measurements were performed on fresh and exposed (for 1 or $2 \mathrm{~h}$ ) solar cells. Figure S4 (left and right) displays partly the results, while Figure S4 (bottom) and Table 2 show the average of the data. The $n$ of PBDB-T:ITIC solar cells remained largely constant around 1.2 over time, indicating there was no increase in trapassisted recombination during light exposure. PBDB-T: [70]PCBM-based cells on average demonstrated an increase in $n$ from 1.43 for fresh devices to about 1.51 after $2 \mathrm{~h}$ of light 

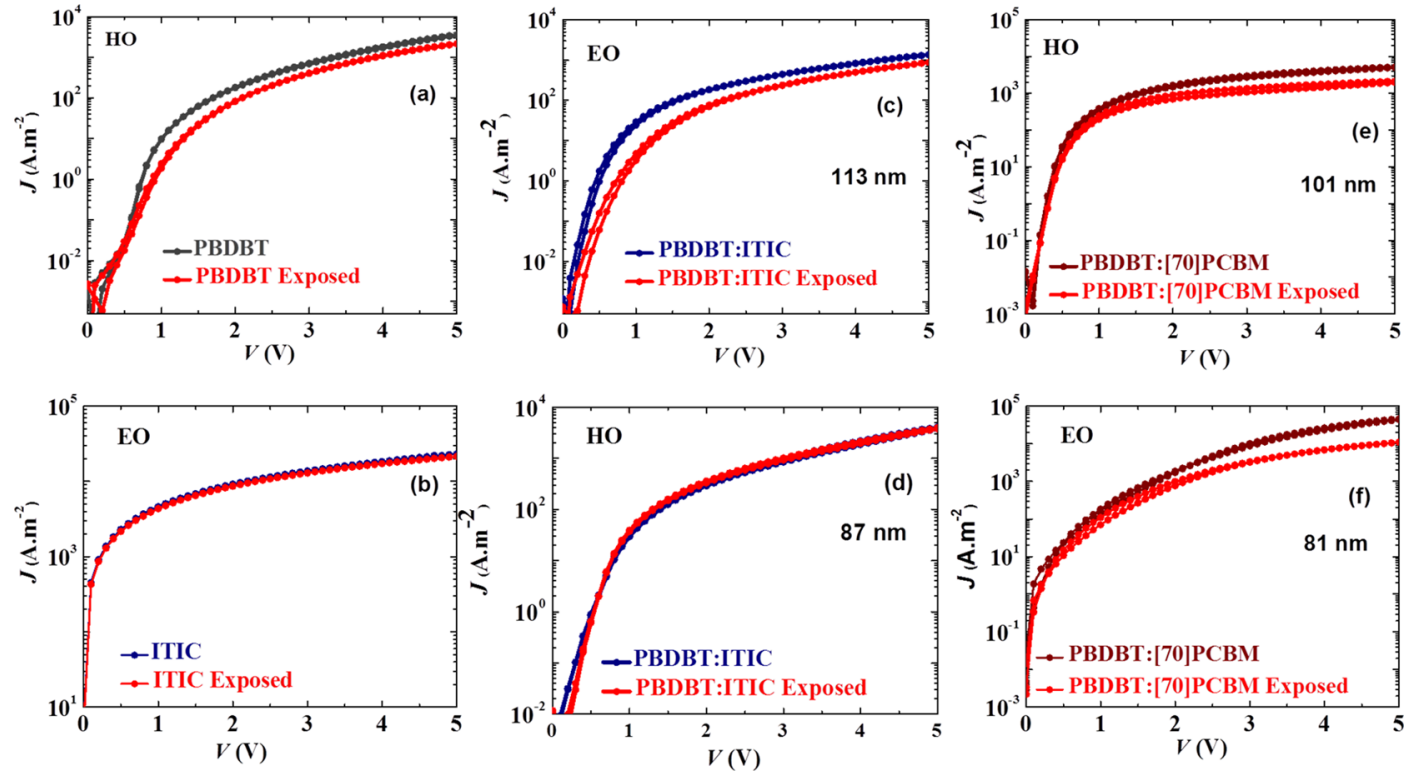

Figure 5. Current-voltage characteristics of fresh and exposed solar cells ( $1 \mathrm{~h}$ - red). Top row: hole-only (HO) devices of PBDB-T (a), PBDBT:ITIC (c), and PBDB-T:[70]PCBM (e). Bottom row: electron-only (EO) devices of ITIC (b), PBDB-T:ITIC (d), and PBDB-T:[70]PCBM (f).

Table 2. Average Mobilities in $10^{-5} \mathrm{~cm}^{2} \mathrm{~V}^{-1} \mathrm{~s}^{-1}$, the Ratio of Mobilities with Corresponding Average Fill Factor (FF), and Average Ideality Factor $(n)$ of Fresh and Exposed ( 1 and 2 h) Solar Cells ${ }^{a}$

\begin{tabular}{|c|c|c|c|c|c|c|c|c|c|c|c|}
\hline PBDB-T device & $\mu_{\mathrm{h}}$ fresh & $\mu_{\mathrm{h}} 1 \mathrm{~h}$ & $\mu_{\mathrm{e}}$ fresh & $\mu_{\mathrm{e}} 1 \mathrm{~h}$ & $\mu_{\max } / \mu_{\min }$ fresh & FF fresh (\%) & $\mu_{\max } / \mu_{\min } 1 \mathrm{~h}$ & FF $1 \mathrm{~h}(\%)$ & $n$ fresh & $n 1 \mathrm{~h}$ & $n 2 \mathrm{~h}$ \\
\hline ITIC & 15 & 17 & 5.1 & 2.8 & 2.9 & 63.9 & 6.1 & 56.8 & 1.19 & 1.20 & 1.19 \\
\hline$[70] \mathrm{PCBM}$ & 10 & 4.5 & 27 & 6.3 & 2.7 & 67.8 & 1.4 & 63.7 & 1.43 & 1.49 & 1.51 \\
\hline
\end{tabular}

${ }^{a} h$ is hole, e is electron, max is maximum, and min is minimum.

exposure, indicating an increase in trap-assisted recombination. Thus, the results point to the fact that the observed degradation in the devices, especially in the FF, is not due to electron traps. If that were to be the case, then ITIC-based devices should not have degraded at all. Thus, though traps may have contributed in the PCE decay of [70]PCBM devices, the main reason behind the differences in degradation pathways of the two types of devices could be related to how balanced the charge mobilities are during light exposure.

To further investigate the origin of reduction of FF under light exposure, we performed transient measurements of recombination and extraction rates $\left(k_{\mathrm{rec}}, k_{\mathrm{ex}}\right)$ in both [70] PCBM- and ITIC-based solar cells to measure the ratio of the rate of recombination to that of extraction $\left(k_{\mathrm{rec}} / k_{\mathrm{ex}}\right)$. It has been shown in the literature that when $k_{\mathrm{rec}} / k_{\mathrm{ex}}$ increases, then the FF decreases. ${ }^{53}$ To measure the recombination rate, we performed TPV measurements under open circuit, using a small perturbation LED light intensity with a step function, which causes an exponential decay of $V_{\mathrm{oc}}$ due to recombination of excess charge carriers. ${ }^{54} \mathrm{~A}$ high input impedance of the oscilloscope (1 M $\Omega$ ) was used to keep the device at open circuit. TPV data of the solar cells are shown in the Supporting Information (Figure S6). The recombination rates of the fresh and the degraded devices at 1 sun, as shown in Table S2 for PBDB-T:ITIC and PBDB-T:[70]PCBM, remained largely constant at different LED light intensities, namely, 0.52 and 0.05 sun. The extraction rates were measured following the experiment described elsewhere. ${ }^{55}$ First, the devices are kept under steady-state conditions at higher light intensity. Then, the light intensity is slightly reduced, while the bias voltage is kept constant, which results in the extraction of extra photogenerated charge carriers. The charge carrier extraction rate is calculated by fitting an exponential function to the decay of the current, carried out under different applied voltages, shown in Figure S5. While the recombination rate stays almost the same, the light exposure (at 1 sun for $2 \mathrm{~h}$ ) causes a reduction in the extraction rates for both types of blends due to a lowering of the mobility of charge carriers (see Table S2). This increases the $k_{\text {rec }} / k_{\text {ex }}$ ratio. For example, $k_{\text {rec }} / k_{\text {ex }}$ (at 0.52 sun) of the PBDB-T:ITIC solar cell increased from 0.114 for the fresh device to 0.15 for the degraded device, while that of the PBDB-T:[70]PCBM solar cell increased from 0.06 to 0.088 . As a consequence of the increase in $k_{\mathrm{rec}} / k_{\mathrm{ex}}, \mathrm{FF}$ is reduced from fresh to degraded devices upon light exposure. The reduction in FF is more pronounced in the case of ITICbased devices, which originates from a larger ratio of $\mu_{\max } / \mu_{\min }$. The highly unbalanced mobilities would cause the formation of the space charges becoming dominant in ITIC devices, as mentioned earlier.

Finally, to validate the obtained results by the transient measurements, the current-voltage curves of the fresh and the degraded devices $(1 \mathrm{~h})$ are fitted using a drift-diffusion simulation. ${ }^{56}$ The fitting procedure consists of (i) scanning of a combination of randomly picked parameters within a reasonable range (see Table S3) and (ii) a fitting procedure optimizing the root mean square (rms) errors of the key performance parameters $J_{\mathrm{sc}}, V_{\mathrm{oc}}$ and FF. All the best fits are shown in Table S4 and have rms errors lower than $1 \%$. Note that only the relevant recombination model parameters, namely, bimolecular recombination and trap-assisted Shockley-Read-Hall recombination, are set as fitting parameters. 

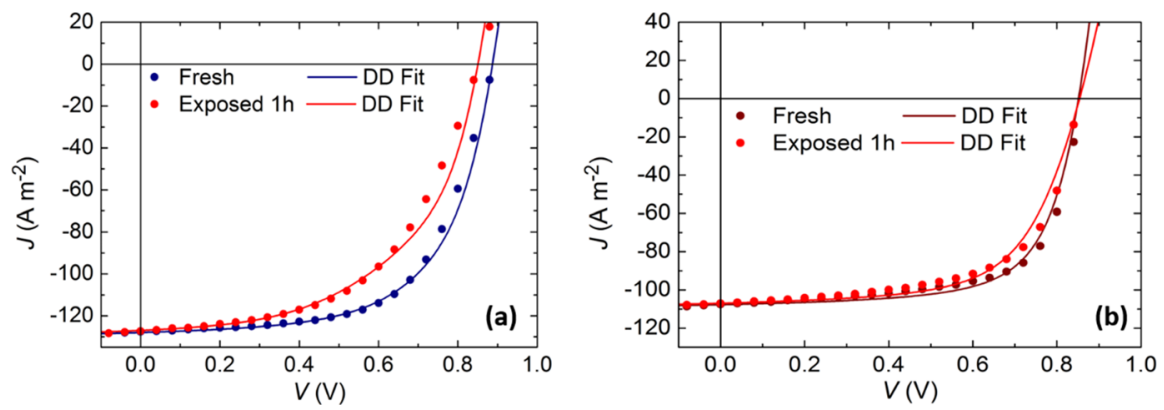

Figure 6. Experimental (dot) and drift-diffusion fitted (line) current-voltage curves for fresh and exposed (1 h) PBDB-T:ITIC (a) and PBDB-T: [70]PCBM (b) solar cells.

The other parameters, such as thicknesses and mobilities, are taken from the experiment.

The fitting in Figure 6 shows that the PBDB-T:ITIC cells are adequately reproduced by only considering bimolecular recombination (see Figure 6a). On the other hand, a small number of traps had to be included to simulate the PBDB-T: [70]PCBM cells properly in Figure 6b. These results are consistent with the measured ideality factors close to 1 for ITIC, indicating that bimolecular recombination is dominant in the ITIC-based devices, and 1.4-1.5 for [70]PCBM, which indicates the presence of both bimolecular and trap-assisted recombinations in this case. From the $V_{\mathrm{oc}}$ light intensity dependence measurements shown in Figure S4, it is concluded that recombination is not the main factor behind the observed degradation. Similarly, all the recombination parameters (see Table S4) do not change much upon exposure, which indicates that the decay of the FF is not due to an increasing amount of recombination. Rather, the extraction rates change with time, pointing to changes in mobilities of the charge carriers. This is also consistent with the almost constant recombination rate obtained by the transient measurements. Also, as concluded from the SCLC and transient measurements, the main parameter responsible for the degradation of the FF is the deterioration of the transport as both electron and hole mobilities decrease upon exposure.

\section{CONCLUSIONS}

The study was designed to assess the role, if any, of the fullerene derivative ([70]PCBM) and non-fullerene (ITIC) acceptors in the photostability of their respective solar cells with PBDB-T (and PTB7-Th). It also envisaged the identification and explanation of the cause(s) of the degradation. The experiments confirmed on the one hand that, though ITIC-based solar cells when blended with PBDB$\mathrm{T}$ performed better in efficiency, they were poor for photostability in comparison to [70]PCBM. On the other hand, ITIC was less efficient and photostable than [70]PCBM when blended with PTB7-Th. These findings indicate that, irrespective of the device structure, the polymer, or the initial efficiency, the [70]PCBM-based devices are more photostable than the ITIC-based ones. We identified the FF as the current-voltage parameter most responsible for the observed photodegradation. We have also shown that trap-assisted recombination cannot be the reason behind the observed photodegradation in the FF, though it could contribute to the degradation of the PCE of the [70]PCBM devices, because the ITIC devices exhibiting the most loss in FF have lower initial traps and do not show any increase in trap-assisted recombination over the time of exposure. Changes in mobilities upon light exposure are identified as the cause in the decay of the FF and as such the main contributor to the observed difference in the photodegradation of the solar cells. Finally, these findings have important implications and contribute to first steps toward the understanding of the stability of fullerene and non-fullerene OSCs. They also contribute toward the understanding of how the issues of stability are more complex than originally assumed: the apparent assumption that NFAs are more stable than FAs may not be entirely true. Also, the findings reveal that complementary absorption should not take precedence in the design rules for the synthesis of new molecules as it appears to be in the case for ITIC. Thus, there is still room for research into organic materials, be it acceptor or donor, that would be both efficient and stable.

\section{EXPERIMENTAL PROCEDURES}

4.1. Device and Film Fabrication. All materials and solvents used in this work are commercially available: the fullerene acceptor, [70]PCBM, is acquired from Solenne BV, the polymers, PBDB-T, and PTB7-Th, and the non-fullerene acceptor, ITIC, are purchased from Solarmer Energy Inc., while anhydrous chlorobenzene (CB) and diiodooctane (DIO) are obtained from Sigma-Aldrich Co. LLB. For single carrier devices and solar cells, the pristine polymer $(15 \mathrm{mg})$ and the blend of the polymer with the acceptor, either with [70]PCBM or with ITIC in a ratio of $1: 1$ with a total weight of $20 \mathrm{mg}$, are dissolved in $1 \mathrm{~mL}$ of anhydrous CB. When necessary, a solvent additive, DIO, is added in $\mathrm{v} / \mathrm{v}$ ratios of $3 \mathrm{vol} \%$ in the case of the blend with [70]PCBM and 0.5 vol \% in the case of ITIC blends. The solutions are stirred overnight on a hot plate and kept at $40{ }^{\circ} \mathrm{C}$. Prepatterned ITO glass (or glass) substrates are successively cleaned with soap water, in deionized water, by acetone and isopropanol in an ultrasonic bath for at least $10 \mathrm{~min}$ in each of the solvents. They are then dried, annealed for at least $10 \mathrm{~min}$ at $140{ }^{\circ} \mathrm{C}$, and treated in a UV-ozone oven for $20 \mathrm{~min}$. Films are fabricated through spin-coating in a glovebox. In the case of the conventional solar cells, a PEDOT:PSS (VP AI4083, H.C. Starck) layer of thickness $50 \mathrm{~nm}$ is first spin-casted in ambient conditions on the cleaned prepatterned ITO glass substrate and subsequently dried at $140{ }^{\circ} \mathrm{C}$ for $10 \mathrm{~min}$ in an oven. For the inverted solar cells, a $\mathrm{ZnO}$ layer of thickness $30 \mathrm{~nm}$ is spincoated from a sol-gel solution, prepared by dissolving zinc acetate $(109.67 \mathrm{mg})$ in 2-methoxyethanol $(1 \mathrm{~mL})$ and ethanolamine $(30.2$ $\mu \mathrm{L})$, atop the ITO substrates and subsequently annealed at $150{ }^{\circ} \mathrm{C}$ for 10-20 min. The blend solutions are then spin-coated atop the PEDOT:PSS layer at $1500 \mathrm{rpm}$ for $5 \mathrm{~s}$ and spin-dried for $60 \mathrm{~s}$ in a glovebox in an inert atmosphere. The spin-coated ITIC-based films are then annealed at 100 (for the conventional cells) or $160{ }^{\circ} \mathrm{C}$ (for the inverted cells) for $10 \mathrm{~min}$. The films are left in vacuum overnight and at $<10^{-7} \mathrm{mbar}$, and the devices eventually are finished by thermal evaporation of $\mathrm{LiF}(1 \mathrm{~nm})$ and $\mathrm{Al}(100 \mathrm{~nm})$ for the conventional cells or $\mathrm{MoO}_{x}(10 \mathrm{~nm})$ and $\mathrm{Al}$ or $\mathrm{Ag}(100 \mathrm{~nm})$ for the inverted cells. The final conventional device structure is ITO/PEDOT:PSS/Blend/LiF/ 
$\mathrm{Al}$ and that of the inverted cell is $\mathrm{ITO} / \mathrm{ZnO} / \mathrm{Blend} / \mathrm{MoO}_{x} / \mathrm{Al}$ (or $\mathrm{Ag})$.

Single carrier devices are fabricated on glass substrates and kept in vacuum overnight. The bottom contacts, $\mathrm{Al}(20 \mathrm{~nm})$ for EO devices and $\mathrm{Cr}(1 \mathrm{~nm}) / \mathrm{Au}(20 \mathrm{~nm})$ for $\mathrm{HO}$ devices, are thermally evaporated at $<10^{-7} \mathrm{mbar}$. The solutions are spin-coated at $1500 \mathrm{rpm}$ for $5 \mathrm{~s}$ and spin-dried for $60 \mathrm{~s}$ atop the substrates. The devices are finished by thermal evaporation of the top contacts with $\mathrm{EO}$ devices having the following structure $\mathrm{Al} / \mathrm{Blend} / \mathrm{LiF} / \mathrm{Al}$ and $\mathrm{HO}$ devices having the following structure $\mathrm{Cr} / \mathrm{Au} / \mathrm{PBDB}-\mathrm{T}$, pristine acceptor, or Blend/Pd/ Au. Finally, films of PBDB-T, pristine acceptor, [70]PCBM, or ITIC and blends are fabricated by spin-coating on glass substrates for UVvis absorption and AFM measurements.

4.2. Characterization. 4.2.1. Current-Voltage Characteristics and UV-Vis Absorption: Current-voltage characteristics of the solar cells and the single carrier devices are taken as previously described. ${ }^{36}$ For the UV-degradation measurement, the cells are continuously exposed to light in an inert atmosphere (with $<0.1 \mathrm{ppm} \mathrm{H}_{2} \mathrm{O}$ and $<0.1$ ppm $\mathrm{O}_{2}$ ) for $2 \mathrm{~h}$ while being kept at $\sim 295 \mathrm{~K}$ by active cooling. In contrast, for light intensity dependence measurement, the cells kept at $\sim 295 \mathrm{~K}$ are exposed to light calibrated with a long-pass filter to 1 sun for $2 \mathrm{~h}$, and the $J-V$ sweeps are recorded with varying light intensity using a set of neutral density filters coupled with the long-pass filter. The absorption measurements are performed on the films in the wavelength range of 300-900 nm with a UV-3600 Shimadzu UVvis-NIR spectrometer against a glass substrate as a reference.

4.2.2. Transient Measurements. For the transient experiments, the samples are illuminated with a biased white light LED with a rise/fall time of $<200 \mathrm{~ns}$ and frequency of $100 \mathrm{~Hz}$, with a pulse width of $5 \mathrm{~ms}$. The rise/fall time of the LED is tested using a photodiode with $<2 \mathrm{~ns}$ rise/ fall time. Subsequent transient signals are acquired using a digital storage oscilloscope (Agilent DSO-X 3034A) with a $350 \mathrm{MHz}$ bandwidth and input resistance of $1 \mathrm{M} \Omega$.

4.2.3. AFM Measurement. The AFM images shown in this paper are obtained as previously described. ${ }^{36}$

\section{ASSOCIATED CONTENT}

\section{S Supporting Information}

The Supporting Information is available free of charge on the ACS Publications website at DOI: 10.1021/acsami.8b20493.

Optical properties, performance of the solar cells, $V_{\text {oc }}$ light intensity dependence studies, TPV and extraction measurements, and drift-diffusion simulation (PDF)

\section{AUTHOR INFORMATION}

\section{Corresponding Authors}

*E-mail: n.y.doumon@rug.nl (N.Y.D.).

*E-mail: 1.j.a.koster@rug.nl (L.J.A.K.).

\section{ORCID $\odot$}

Nutifafa Y. Doumon: 0000-0002-2625-1647

Azadeh Rahimi Chatri: 0000-0002-2312-1425

L. Jan Anton Koster: 0000-0002-6558-5295

\section{Author Contributions}

Experiments are designed by N.Y.D. and L.J.A.K. Experiments are performed by N.Y.D., M.V.D., F.V.H., and A.R.C. Simulation work is done by V.M.L.C. and L.J.A.K. The manuscript is written by N.Y.D. through contributions from V.M.L.C., A.R.C., and L.J.A.K. All authors have approved the final version of the manuscript.

\section{Funding}

The work is funded by the Zernike Bonus Incentive Scheme Grant and supported by a grant from STW/NWO (VIDI 13476).

\section{Notes}

The authors declare no competing financial interest.

\section{ACKNOWLEDGMENTS}

This work is part of the research program of the Foundation of Fundamental Research on Matter (FOM), which is part of The Netherlands Organisation for Scientific Research (NWO). This is a publication of the FOM-focus Group "Next Generation Organic Photovoltaics", participating in the Dutch Institute for Fundamental Energy Research (DIFFER). The work is funded by the Zernike Bonus Incentive Scheme Grant. N.Y.D. would like to acknowledge Arjen Kamp and Teodor Zaharia for their technical support. The work of L.J.A.K., V.M.L.C., and A.R.C. is supported by a grant from STW/NWO (VIDI 13476).

\section{REFERENCES}

(1) Abbaszadeh, D.; Doumon, N. Y.; Wetzelaer, G. J. A. H.; Koster, L. J. A.; Blom, P. W. M. Effect of the Layer Thickness on the Efficiency Enhancement in Bilayer Polymer Light-Emitting Diodes. Synth. Met. 2016, 215, 64-67.

(2) Cui, C.; Wong, W.-Y.; Li, Y. Improvement of Open-Circuit Voltage and Photovoltaic Properties of 2D-Conjugated Polymers by Alkylthio Substitution. Energy Environ. Sci. 2014, 7, 2276-2284.

(3) Shi, G.; Yuan, J.; Huang, X.; Lu, Y.; Liu, Z.; Peng, J.; Ding, G.; Shi, S.; Sun, J.; Lu, K.; et al. Combinative Effect of Additive and Thermal Annealing Processes Delivers High Efficiency All-Polymer Solar Cells. J. Phys. Chem. C 2015, 119, 25298-25306.

(4) Sun, C.; Wu, Z.; Hu, Z.; Xiao, J.; Zhao, W.; Li, H.-W.; Li, Q.-Y.; Tsang, S.-W.; Xu, Y.-X.; Zhang, K.; et al. Interface Design for HighEfficiency Non-Fullerene Polymer Solar Cells. Energy Environ. Sci. 2017, 10, 1784-1791.

(5) Xie, Y.; Hu, X.; Yin, J.; Zhang, L.; Meng, X.; Xu, G.; Ai, Q.; Zhou, W.; Chen, Y. Butanedithiol Solvent Additive Extracting Fullerenes from Donor Phase To Improve Performance and Photostability in Polymer Solar Cells. ACS Appl. Mater. Interfaces 2017, 9, 9918-9925.

(6) You, J.; Dou, L.; Yoshimura, K.; Kato, T.; Ohya, K.; Moriarty, T.; Emery, K.; Chen, C.-C.; Gao, J.; Li, G.; et al. A Polymer Tandem Solar Cell with $10.6 \%$ Power Conversion Efficiency. Nat. Commun. 2013, 4, 1446.

(7) Zhang, S.; Ye, L.; Hou, J. Breaking the 10\% Efficiency Barrier in Organic Photovoltaics: Morphology and Device Optimization of Well-Known PBDTTT Polymers. Adv. Energy Mater. 2016, 6, 1502529.

(8) Fan, B.; Ying, L.; Wang, Z.; He, B.; Jiang, X.-F.; Huang, F.; Cao, Y. Optimisation of Processing Solvent and Molecular Weight for the Production of Green-Solvent-Processed All-Polymer Solar Cells with a Power Conversion Efficiency over 9\%. Energy Environ. Sci. 2017, 10, $1243-1251$.

(9) Graetzel, M.; Janssen, R. A. J.; Mitzi, D. B.; Sargent, E. H. Materials Interface Engineering for Solution-Processed Photovoltaics. Nature 2012, 488, 304-312.

(10) Guo, F.; Karl, A.; Xue, Q.-F.; Tam, K. C.; Forberich, K.; Brabec, C. J. The Fabrication of Color-Tunable Organic Light-Emitting Diode Displays via Solution Processing. Light Sci. Appl. 2017, 6, No. e17094.

(11) Guo, X.; Cui, C.; Zhang, M.; Huo, L.; Huang, Y.; Hou, J.; Li, Y. High Efficiency Polymer Solar Cells Based on Poly(3-Hexylthiophene)/Indene-C70 Bisadduct with Solvent Additive. Energy Environ. Sci. 2012, 5, 7943.

(12) Huang, J.; Li, C. Z.; Chueh, C. C.; Liu, S. Q.; Yu, J. S.; Jen, A K. Y. $10.4 \%$ Power Conversion Efficiency of ITO-Free Organic Photovoltaics Through Enhanced Light Trapping Configuration. Adv. Energy Mater. 2015, 5, 1500406.

(13) Kan, B.; Zhang, Q.; Li, M.; Wan, X.; Ni, W.; Long, G.; Wang, Y.; Yang, X.; Feng, H.; Chen, Y. Solution-Processed Organic Solar Cells Based on Dialkylthiol-Substituted Benzodithiophene Unit with Efficiency near 10\%. J. Am. Chem. Soc. 2014, 136, 15529-15532.

(14) Yusoff, A. R. B. M.; Kim, D.; Kim, H. P.; Shneider, F. K.; da Silva, W. J.; Jang, J. A High Efficiency Solution Processed Polymer 
Inverted Triple-Junction Solar Cell Exhibiting a Power Conversion Efficiency of 11.83\%. Energy Environ. Sci. 2015, 8, 303-316.

(15) Qin, Y.; Chen, Y.; Cui, Y.; Zhang, S.; Yao, H.; Huang, J.; Li, W.; Zheng, Z.; Hou, J. Achieving $12.8 \%$ Efficiency by Simultaneously Improving Open-Circuit Voltage and Short-Circuit Current Density in Tandem Organic Solar Cells. Adv. Mater. 2017, 29, 1606340.

(16) Chen, J.-D.; Cui, C.; Li, Y.-Q.; Zhou, L.; Ou, Q.-D.; Li, C.; Li, Y.; Tang, J.-X. Single-Junction Polymer Solar Cells Exceeding 10\% Power Conversion Efficiency. Adv. Mater. 2015, 27, 1035-1041.

(17) Liao, S.-H.; Jhuo, H.-J.; Yeh, P.-N.; Cheng, Y.-S.; Li, Y.-L.; Lee, Y.-H.; Sharma, S.; Chen, S.-A. Single Junction Inverted Polymer Solar Cell Reaching Power Conversion Efficiency $10.31 \%$ by Employing Dual-Doped Zinc Oxide Nano-Film as Cathode Interlayer. Sci. Rep. 2014, 4, 6813.

(18) Liu, C.; Yi, C.; Wang, K.; Yang, Y.; Bhatta, R. S.; Tsige, M.; Xiao, S.; Gong, X. Single-Junction Polymer Solar Cells with Over 10\% Efficiency by a Novel Two-Dimensional Donor-Acceptor Conjugated Copolymer. ACS Appl. Mater. Interfaces 2015, 7, 4928-4935.

(19) Xia, Y.; Musumeci, C.; Bergqvist, J.; Ma, W.; Gao, F.; Tang, Z.; Bai, S.; Jin, Y.; Zhu, C.; Kroon, R.; et al. Inverted All-Polymer Solar Cells Based on a Quinoxaline-thiophene/Naphthalene-Diimide Polymer Blend Improved by Annealing. J. Mater. Chem. A 2016, 4, $3835-3843$

(20) Yuan, J.; Ma, W. High Efficiency All-Polymer Solar Cells Realized by the Synergistic Effect between the Polymer Side-Chain Structure and Solvent Additive. J. Mater. Chem. A 2015, 3, 70777085

(21) Xu, Y.; Yuan, J.; Sun, J.; Zhang, Y.; Ling, X.; Wu, H.; Zhang, G.; Chen, J.; Wang, Y.; Ma, W. Widely Applicable N-Type Molecular Doping for Enhanced Photovoltaic Performance of All-Polymer Solar Cells. ACS Appl. Mater. Interfaces 2018, DOI: 10.1021/acsami.7b 15000

(22) Fan, B.; Ying, L.; Zhu, P.; Pan, F.; Liu, F.; Chen, J.; Huang, F.; Cao, Y. All-Polymer Solar Cells Based on a Conjugated Polymer Containing Siloxane-Functionalized Side Chains with Efficiency over 10\%. Adv. Mater. 2017, 29, 1703906.

(23) An, M.; Xie, F.; Geng, X.; Zhang, J.; Jiang, J.; Lei, Z.; He, D.; Xiao, Z.; Ding, L. A High-Performance D-A Copolymer Based on Dithieno[3,2-b:2",3"-d]Pyridin-5(4H)-One Unit Compatible with Fullerene and Nonfullerene Acceptors in Solar Cells. Adv. Energy Mater. 2017, 7, 1602509.

(24) Firdaus, Y.; Maffei, L. P.; Cruciani, F.; Müller, M. A.; Liu, S.; Lopatin, S.; Wehbe, N.; Ndjawa, G. O. N.; Amassian, A.; Laquai, F.; et al. Polymer Main-Chain Substitution Effects on the Efficiency of Nonfullerene BHJ Solar Cells. Adv. Energy Mater. 2017, 7, 1700834.

(25) Gasparini, N.; Salvador, M.; Strohm, S.; Heumueller, T.; Levchuk, I.; Wadsworth, A.; Bannock, J. H.; de Mello, J. C.; Egelhaaf, H. J.; Baran, D.; et al. Burn-in Free Nonfullerene-Based Organic Solar Cells. Adv. Energy Mater. 2017, 7, 1700770.

(26) Li, S.; Ye, L.; Zhao, W.; Zhang, S.; Mukherjee, S.; Ade, H.; Hou, J. Energy-Level Modulation of Small-Molecule Electron Acceptors to Achieve over $12 \%$ Efficiency in Polymer Solar Cells. Adv. Mater. 2016, 28, 9423-9429.

(27) Liu, X.; Xie, B.; Duan, C.; Wang, Z.; Fan, B.; Zhang, K.; Lin, B.; Colberts, F. J. M.; Ma, W.; Janssen, R. A. J.; et al. A High Dielectric Constant Non-Fullerene Acceptor for Efficient Bulk-Heterojunction Organic Solar Cells. J. Mater. Chem. A 2018, 395-403.

(28) Yu, T.; Xu, X.; Zhang, G.; Wan, J.; Li, Y.; Peng, Q. Wide Bandgap Copolymers Based on Quinoxalino[6,5-f].Quinoxaline for Highly Efficient Nonfullerene Polymer Solar Cells. Adv. Funct. Mater. 2017, 27, 1701491.

(29) Zhao, W.; Qian, D.; Zhang, S.; Li, S.; Inganäs, O.; Gao, F.; Hou, J. Fullerene-Free Polymer Solar Cells with over 11\% Efficiency and Excellent Thermal Stability. Adv. Mater. 2016, 4734-4739.

(30) Li, S.; Ye, L.; Zhao, W.; Yan, H.; Yang, B.; Liu, D.; Li, W.; Ade, H.; Hou, J. A Wide Band Gap Polymer with a Deep Highest Occupied Molecular Orbital Level Enables 14.2\% Efficiency in Polymer Solar Cells. J. Am. Chem. Soc. 2018, 140, 7159-7167.
(31) Zhang, S.; Qin, Y.; Zhu, J.; Hou, J. Over 14\% Efficiency in Polymer Solar Cells Enabled by a Chlorinated Polymer Donor. Adv. Mater. 2018, 30, 1800868.

(32) Zheng, Z.; Hu, Q.; Zhang, S.; Zhang, D.; Wang, J.; Xie, S.; Wang, R.; Qin, Y.; Li, W.; Hong, L.; et al. A Highly Efficient NonFullerene Organic Solar Cell with a Fill Factor over 0.80 Enabled by a Fine-Tuned Hole-Transporting Layer. Adv. Mater. 2018, 30, 1801801.

(33) Meng, L.; Zhang, Y.; Wan, X.; Li, C.; Zhang, X.; Wang, Y.; Ke, X.; Xiao, Z.; Ding, L.; Xia, R.; et al. Organic and Solution-Processed Tandem Solar Cells with 17.3\% Efficiency. Science 2018, 361, 10941098.

(34) Zhang, G.; Zhao, J.; Chow, P. C. Y.; Jiang, K.; Zhang, J.; Zhu, Z.; Zhang, J.; Huang, F.; Yan, H. Nonfullerene Acceptor Molecules for Bulk Heterojunction Organic Solar Cells. Chem. Rev. 2018, 118, 3447-3507.

(35) Domínguez, I. F.; Topham, P. D.; Bussière, P. O.; Bégué, D.; Rivaton, A. Unravelling the Photodegradation Mechanisms of a Low Bandgap Polymer by Combining Experimental and Modeling Approaches. J. Phys. Chem. C 2015, 119, 2166-2176.

(36) Doumon, N. Y.; Wang, G.; Chiechi, R. C.; Koster, L. J. A. Relating Polymer Chemical Structure to the Stability of Polymer:Fullerene Solar Cells. J. Mater. Chem. C 2017, 5, 6611-6619.

(37) Inasaridze, L. N.; Shames, A. I.; Martynov, I. V.; Li, B.; Mumyatov, A. V.; Susarova, D. K.; Katz, E. A.; Troshin, P. A. LightInduced Generation of Free Radicals by Fullerene Derivatives: An Important Degradation Pathway in Organic Photovoltaics? J. Mater. Chem. A 2017, 5, 8044-8050.

(38) Jacobs, I. E.; Wang, F.; Bedolla Valdez, Z. I.; Ayala Oviedo, A. N.; Bilsky, D. J.; Moulé, A. J. Photoinduced Degradation from Trace 1,8-Diiodooctane in Organic Photovoltaics. J. Mater. Chem. C 2018, 6 , 219.

(39) Kesters, J.; Verstappen, P.; Raymakers, J.; Vanormelingen, W.; Drijkoningen, J.; D’Haen, J.; Manca, J.; Lutsen, L.; Vanderzande, D.; Maes, W. Enhanced Organic Solar Cell Stability by Polymer (PCPDTBT) Side Chain Functionalization. Chem. Mater. 2015, 27, $1332-1341$.

(40) Salvador, M.; Gasparini, N.; Perea, J. D.; Paleti, S. H.; Distler, A.; Inasaridze, L. N.; Troshin, P. A.; Lüer, L.; Egelhaaf, H.-J.; Brabec, C. Suppressing Photooxidation of Conjugated Polymers and Their Blends with Fullerenes through Nickel Chelates. Energy Environ. Sci. 2017, 10, 2005-2016.

(41) Savagatrup, S.; Printz, A. D.; O’Connor, T. F.; Zaretski, A. V.; Rodriquez, D.; Sawyer, E. J.; Rajan, K. M.; Acosta, R. I.; Root, S. E.; Lipomi, D. J. Mechanical Degradation and Stability of Organic Solar Cells: Molecular and Microstructural Determinants. Energy Environ. Sci. 2015, 8, 55-80.

(42) Tournebize, A.; Rivaton, A.; Gardette, J. L.; Lombard, C.; Pépin-Donat, B.; Beaupré, S.; Leclerc, M. How Photoinduced Crosslinking under Operating Conditions Can Reduce PCDTBTBased Solar Cell Efficiency and Then Stabilize It. Adv. Energy Mater. 2014, 4, 1301530.

(43) Tremolet de Villers, B. J.; O’Hara, K. A.; Ostrowski, D. P.; Biddle, P. H.; Shaheen, S. E.; Chabinyc, M. L.; Olson, D. C.; Kopidakis, N. Removal of Residual Diiodooctane Improves Photostability of High-Performance Organic Solar Cell Polymers. Chem. Mater. 2016, 28, 876-884.

(44) Zhang, Y.; Xu, Y.; Ford, M. J.; Li, F.; Sun, J.; Ling, X.; Wang, Y.; Gu, J.; Yuan, J.; Ma, W. Thermally Stable All-Polymer Solar Cells with High Tolerance on Blend Ratios. Adv. Energy Mater. 2018, 8, 1800029.

(45) Holliday, S.; Ashraf, R. S.; Wadsworth, A.; Baran, D.; Yousaf, S. A.; Nielsen, C. B.; Tan, C.-H.; Dimitrov, S. D.; Shang, Z.; Gasparini, N.; et al. High-Efficiency and Air-Stable P3HT-Based Polymer Solar Cells with a New Non-Fullerene Acceptor. Nat. Commun. 2016, 7, 11585 .

(46) Baran, D.; Ashraf, R. S.; Hanifi, D. A.; Abdelsamie, M.; Gasparini, N.; Röhr, J. A.; Holliday, S.; Wadsworth, A.; Lockett, S.; Neophytou, M.; et al. Reducing the Efficiency-Stability-Cost Gap of Organic Photovoltaics with Highly Efficient and Stable Small 
Molecule Acceptor Ternary Solar Cells. Nat. Mater. 2017, 16, 363369.

(47) Baran, D.; Gasparini, N.; Wadsworth, A.; Tan, C. H.; Wehbe, N.; Song, X.; Hamid, Z.; Zhang, W.; Neophytou, M.; Kirchartz, T.; et al. Robust Nonfullerene Solar Cells Approaching Unity External Quantum Efficiency Enabled by Suppression of Geminate Recombination. Nat. Commun. 2018, 2059.

(48) Cha, H.; Wu, J.; Wadsworth, A.; Nagitta, J.; Limbu, S.; Pont, S.; Li, Z.; Searle, J.; Wyatt, M. F.; Baran, D.; et al. An Efficient, "Burn in" Free Organic Solar Cell Employing a Nonfullerene Electron Acceptor. Adv. Mater. 2017, 29, 1701156.

(49) Kim, W.; Kim, J. K.; Kim, E.; Ahn, T. K.; Wang, D. H.; Park, J. H. Conflicted Effects of a Solvent Additive on PTB7:PC71BM Bulk Heterojunction Solar Cells. J. Phys. Chem. C 2015, 119, 5954-5961.

(50) Pearson, A. J.; Hopkinson, P. E.; Couderc, E.; Domanski, K.; Abdi-Jalebi, M.; Greenham, N. C. Critical Light Instability in CB/ DIO Processed PBDTTT-EFT:PC71BM Organic Photovoltaic Devices. Org. Electron. 2016, 30, 225-236.

(51) Yao, H.; Cui, Y.; Yu, R.; Gao, B.; Zhang, H.; Hou, J. Design, Synthesis, and Photovoltaic Characterization of a Small Molecular Acceptor with an Ultra-Narrow Band Gap. Angew. Chem., Int. Ed. 2017, 56, 3045-3049.

(52) Mandoc, M. M.; Kooistra, F. B.; Hummelen, J. C.; De Boer, B.; Blom, P. W. M. Effect of Traps on the Performance of Bulk Heterojunction Organic Solar Cells. Appl. Phys. Lett. 2007, 91, 263505.

(53) Bartesaghi, D.; Pérez, I. D. C.; Kniepert, J.; Roland, S.; Turbiez, M.; Neher, D.; Koster, L. J. A. Competition between Recombination and Extraction of Free Charges Determines the Fill Factor of Organic Solar Cells. Nat. Commun. 2015, 6, 7083.

(54) Rahimi Chatri, A.; Torabi, S.; Le Corre, V. M.; Koster, L. J. A. Impact of Electrodes on Recombination in Bulk Heterojunction Organic Solar Cells. ACS Appl. Mater. Interfaces 2018, 10, 12013.

(55) Le Corre, V. M.; Chatri, A. R.; Doumon, N. Y.; Koster, L. J. A. Charge Carrier Extraction in Organic Solar Cells Governed by SteadyState Mobilities. Adv. Energy Mater. 2017, 7, 1701138.

(56) Koster, L. J. A.; Smits, E. C. P.; Mihailetchi, V. D.; Blom, P. W. M. Device Model for the Operation of Polymer/Fullerene Bulk Heterojunction Solar Cells. Phys. Rev. B 2005, 72, 085205. 